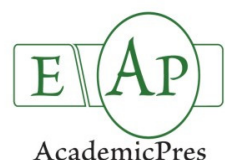

AcademicPres

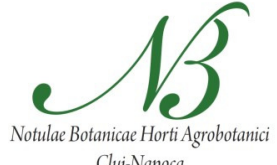

Cluj-Napoca

\title{
Genetic Similarity Assessment among Selected Naked Oat Cultivars and Breeding Lines Using ISSR Markers
}

\author{
Edyta PACZOS-GRZEDA ${ }^{1 *}$, Piotr Tomasz BEDNAREK ${ }^{2}$, Aneta KOROLUK ${ }^{1}$, \\ Zygmunt NITA ${ }^{3}$, Zofia BANASZAK ${ }^{4}$, Andrzej BICHONSKI ${ }^{5}$, Marek CHMIEL ${ }^{5}$, \\ Agnieszka GRADZIELEWSKA ${ }^{1}$, Katarzyna NOWACZYK ${ }^{4}$, Aleksandra \\ SZOLKOWSKA ${ }^{4}$, Krystyna WERWINSKA ${ }^{3}$, Patrycja WIECZOREK ${ }^{5}$ \\ ${ }^{1}$ University of Life Sciences in Lublin, Institute of Plant Genetics, Breeding and Biotechnology, Akademicka Str 15, 20-950 Lublin, Poland; \\ edyta.paczos@up.lublin.pl(*correspondingauthor); aneta.koroluk@up.lublin.pl;agnieszka.gradzielewska@up.lublin.pl \\ ${ }^{2}$ Plant Breeding and Acclimatization Institute-NRI, Radzikow,05-870Blonie,Poland;p.bednarek@ihar.edu.pl \\ ${ }^{3}$ Strzelce Plant Breeding Company, Glowna Str 20,99-307 Strzelce,Poland; z_nita@hr-strzelce.pl;k_werwinska@hr-strzelce.pl \\ ${ }^{4}$ DANKO Plant Breeding Company,Choryn 27,64-000Koscian,Poland; zofia.banaszak@danko.pl;katarzyna.nowaczyk@danko.pl, \\ aleksandra.szolkowska@danko.pl \\ ${ }^{5}$ Malopolska Plant BreedingCompany,Polanowice,32-090Slomniki,Poland;andrzejb@bbp.pl;jare-polanowice@wp.pl;patrycjawieczorek19@interia.pl
}

\begin{abstract}
Naked oat refers to a variety of Avena sativa with lemma and palea separating from the grains. Its spikelets are multiflorous and morphologically different from the husked oat. Problems with preharvest sprouting, threshability, rancidity, a wide range of kernel sizes, as well as its relatively low tolerance to limited soil water content, are its main drawbacks. Nevertheless it could be an alternative to a conventional oat. Unfortunately, its genetic variation is still poorly recognized. In the given study a set of 26 naked oat cultivars and lines were analyzed with 25 inter-simple sequence repeats (ISSR) primers that amplified as many as 429 DNA fragments among which 204 were polymorphic. The average number of markers amplified per primer pair and polymorphism information content (PIC) value equaled to eight and 0.23 , respectively. Forty four unique PCR products were identified for different genotypes. While Unweighted Pair-Group Method with Arithmetic Mean failed to distinguish the materials into main clusters it demonstrated that cultivars 'Akt', 'Polar', 'Cacko', 'Siwek', 'Nagus' and most of the DC lines were within a single group. Moreover, the cultivars that were closely related based on their breeding pedigree (related to 'Akt') were close to each other. Principal Coordinate Analysis explained 54.1\% of variance and was in good agreement with the UPGMA. ISSR markers could be used for the evaluation of genetic similarity of cultivars and lines as well as the differentiation of individual genotypes. This study demonstrated that the available $A$. sativa naked type genetic pool is relatively wide and have the potential for further breeding progress.
\end{abstract}

Keywords: Avena sativa, genetic pool, molecular markers, genetic diversity

\section{Introduction}

Common oat is a cereal crop adapted to moderate climate, cultivated predominantly in temperate regions (Burrows, 1986). It is used for forage, feed and food production. Varieties of cultivated oat belong to Avena sativa ssp. sativa L. (spring oat, naked and husked type) (Valentine, 1995), and A. sativa ssp. byzantina L. (red oat) (Zeller, 1998).

High quality groat with excellent fat and amino acid composition and husk having an energy yield like that of straw determines the nutritional value of conventional, covered oat. Due to the husk, the yield of total energy is weaker in oat than in case of other cereals. Thus, the cultivation and use of oat is behind other plants rich in energy (Kirkkari, 2008). Moreover, husk decreases bulk density, reducing storage and transport efficiency (Burrows, 1986). Its percentage ranges from a minimum of $21 \%$ to a maximum of $41 \%$ (Ronald et al., 1999). Husks comprise mainly of cell wall (> 83\%) with nearly equal quantities of cellulose and hemicellulose (30-35\%) (Welch et al., 1983). Protein and oil contents are low (1.6-5\% and 1-2.2\%) (Salo and Kotilainen, 1970). Starch and water-soluble carbohydrates contents do not exceed $2 \%$ and $1 \%$, respectively (Welch et al., 1983). Husk composition within and among varieties may vary due to location and weather conditions (Ronald et al., 1997; Sykut-Domańska, 2012). Oat husk is a poor quality feedstuff. The improvement in 
grain quality could be achieved by breeding for reduced husk content or breeding high yielding naked oat cultivars (Kirkkari, 2008).

Naked oat refers to a variety with lemma and palea separating from the grains during threshing. The spikelets of naked oat are multiflorous and morphologically different from the husked oat (Valentine, 1995). Whereas, husked oat spikelets may contain 1 to 3 (rarely 4) florets, naked oat consists of 4 to 7 , but quite often may possess up to 12 florets (Burrows, 1986; Valentine, 1995). This trait, mostly controlled by a single dominant gene, is modified by several minor loci (Valentine, 1995; Doehlert et al., 2006). The oat kernels are covered with fine hair termed trichomes. Trichomes are problematic, and efforts are being made in order to obtain cultivars with reduced pubescence (Kirkkari et al., 2009). Existed differences of cultivars in pubescence resulted in attempts of breeding varieties with reduced trichome numbers.

Naked oat cultivars are characterized by lower mass of thousand grain and higher hectolitre weight than common oat (Sykut-Domańska, 2012). The yield of naked oats is ca. $25-30 \%$ lower than the yield of the common one. However, when the same characteristics are recalculated for seeds without husk, the results are comparable (Nita, 2003). Moreover, naked oat is enriched in fat and unsaturated fatty acids, water - soluble $\beta$ - glucans forming dietary fibre and good quality proteins (Welch et al., 1983). It should be mentioned that the chemical content of naked oat seeds may vary significantly on different continents. Canadian and the USA varieties have more proteins ( $>14 \%)$ and fewer fats $(<6 \%)$ while European and Australian forms have fewer proteins (11-13\%) but are richer in fats $(7-10 \%)$ (Nita 2003). Unfortunately, problems with preharvest sprouting, threshability, rancidity, a wide range of kernel sizes as well as its relatively low tolerance to limited soil water content (Nita, 2003) are its main drawbacks (Forsberg and Reeves, 1992). Moreover, the husk plays a crucial role in protecting the groat from damage (Burrows, 1986) and pathogenic attack (Ronald et al., 1999). Nevertheless, dehusked conventional oat, does not achieve comparable economic result as naked one, as dehusking costs and disposition of the husk waste is considerably large (Kirkkari, 2008). Naked oat could be a suitable alternative to conventional oat.

There is an interest in the evaluation of genetic diversity of the available oat genetic pool and use of this information in practical breeding (Tinker et al., 2009). Genetic diversity of breeding materials depends largely on differentiation of parental lines used for crossing in conventional breeding programs during last decades. Moreover, selection directed to create improved locally adapted crop varieties, tends to erode genetic diversity. For pragmatic reasons, most oat breeders favour crosses among their own best varieties, which guarantees short-term success. Molecular studies using AFLP markers applied to a core set of cultivated oat germplasm (Fu et al., 2005), as well as analyses performed with DArTs (Tinker et al., 2009), indicated that most genetic relatedness was associated with geographical origin and development within the same breeding program. The evaluation of genetic diversity plays a prominent role in the characterization of breeding lines or varieties. It could be also applied for the selection of parents in the development of crossing schemes (Paczos-Grzeda, 2004). The higher genetic distance between parental forms, the greater probability to achieve new allele combinations (Cox and Murphy, 1990). Better knowledge of genetic relatedness of parents selected for crossing could help to maintain genetic diversity, evaluate the potential vulnerability to abiotic stresses and pests, sustain long-term selection gain and assure continued genetic improvement (Martin et al., 1991; Chowdhury et al., 2002). Therefore, understanding the genetic relationships among naked oat breeding lines, could maximize the rational use of the genetic resources and encourage breeders to use new germplasm. The primary focus of the study was to perform preliminary estimation of the available genetic pool of the naked oat cultivars registered in Poland and 18 breeding lines based on ISSR marker polymorphisms.

\section{Materials and Methods}

\section{Plant material and DNA extractions}

Twenty six naked oats (A. sativa L.) including five cultivars registered in Poland (cv. 'Akt' was represented by four sublines) and eighteen breeding lines from three breeding programs listed in Tab. 1 were analysed. Pedigree of oat cultivars was reproduced based on breeding records. Genomic DNA was extracted from 15-20 coleoptiles of several day old seedlings of each breeding form following the CTAB procedure (Doyle and Doyle, 1987).

Tab. 1. Arrangement of the analysed naked oat cultivars and lines with their descriptive data. D, M, S states for breeding programs run in commercial companies

\begin{tabular}{|c|c|c|c|}
\hline $\begin{array}{l}\text { Cultivar/ } \\
\text { Line }\end{array}$ & Pedigree & $\begin{array}{l}\text { Breeding } \\
\text { program }\end{array}$ & Comments \\
\hline 'Akt' & 'Adam'×‘Adamo' & $S$ & Registered in 1997 \\
\hline 'Akt' 1 & 'Adam’×‘Adamo' & $S$ & $\begin{array}{l}\text { Sister line of cv. } \\
\text { 'Akt' }\end{array}$ \\
\hline 'Akt' 2 & 'Adam'×'Adamo' & $S$ & $\begin{array}{l}\text { Sister line of cv. } \\
\text { 'Akt' }\end{array}$ \\
\hline 'Akt' bis & 'Adam'×‘Adamo' & $S$ & $\begin{array}{l}\text { Sister line of cv. } \\
\text { 'Akt' }\end{array}$ \\
\hline STH 721 & 'Akt'×AVE 2999 & S & $\begin{array}{c}\text { AVE } 2999-A . \\
\text { sterilis }\end{array}$ \\
\hline 'Polar' & 'Ago'×'Ramiro' & S & Registered in 2002 \\
\hline 'Cacko’ & 'Ago'×'Ramiro' & S & Registered in 2000 \\
\hline 'Maczo' & STH7520×STH7376 & $S$ & Registered in 2010 \\
\hline STH 8307 & STH $8070 \times$ STH 6102 & S & Preliminary field trial \\
\hline STH 6315 & STH $14063 \times$ STH 13876 & $S$ & Preliminary field trial \\
\hline 'Siwek' & 'Benquel' × 'Akt' & M & Registered in 2010 \\
\hline 'Nagus' & 'Auron'×POB-W 481 & $\mathrm{D}$ & Registered in 2011 \\
\hline DC 2188 & STH 3997x‘Jakub’ & $\mathrm{D}$ & Preliminary field trial \\
\hline DC 2215 & 'Bullion'×'Mozart' & $\mathrm{D}$ & Preliminary field trial \\
\hline DC 2612 & 'Samuel'×STH 6202 & $\mathrm{D}$ & Preliminary field trial \\
\hline DC 2711 & ('Izak'×'Bullion')x 'Akt' & $\mathrm{D}$ & Preliminary field trial \\
\hline DC 2973 & 'Polar'×'Mozart' & $\mathrm{D}$ & Preliminary field trial \\
\hline DC 3674 & STH 4899×'Polar’ & $\mathrm{D}$ & Preliminary field trial \\
\hline DC 3080 & $\mathrm{P}(508) 19251 \times \mathrm{CHD} 1743$ & $\mathrm{D}$ & Preliminary field trial \\
\hline DC 3996 & $\mathrm{P}(508) 19251 \times \mathrm{CHD} 1743$ & $\mathrm{D}$ & Preliminary field trial \\
\hline DC 3416 & 'Sallust'×‘Akt' & $\mathrm{D}$ & Preliminary field trial \\
\hline DC 3249 & POB-W 481×‘Jawor’ & $\mathrm{D}$ & Preliminary field trial \\
\hline DC 3170 & $\mathrm{P}(508) 19251 \times \mathrm{CHD} 1743$ & $\mathrm{D}$ & Preliminary field trial \\
\hline DC 2567 & ‘Auron’×POB-W 481 & $\mathrm{D}$ & Preliminary field trial \\
\hline DC 2931 & STH $4879 \times$ ×Sallust' & $\mathrm{D}$ & Preliminary field trial \\
\hline DC 2934 & STH 4879×'Sallust' & $\mathrm{D}$ & Preliminary field trial \\
\hline
\end{tabular}


68

\section{ISSR}

Amplification was performed according to the ISSR method described by Zietkiewicz et al., (1994) with modifications. Reaction mixture of $15 \mu$ l contained $1 \times$ PCR Buffer $(10 \mathrm{mM}$ Tris pH 8.8; $50 \mathrm{mM} \mathrm{KCl}$; $0.08 \%$ Nonidet P40), $160 \mu \mathrm{M}$ of each dNTP, $0.35 \mathrm{pmol}$ of primer, $1.3 \mathrm{mM}$ of $\mathrm{MgCl}_{2}, 0.4 \mathrm{mM}$ of spermidine, $0.5 \mathrm{U}$ of Taq DNA Polymerase and $30 \mathrm{ng}$ of template DNA. The twenty five ISSR random primers were used in the analysis. Sequences of the applied primers are presented in Tab. 2. Amplifications were carried out in a T1 Biometra thermal cycler with an initial denaturation step at $95^{\circ} \mathrm{C}$ for $4 \mathrm{~min}$, followed by 3 cycles of $30 \mathrm{sec}$ at $95^{\circ} \mathrm{C}, 45 \mathrm{sec}$ at $54^{\circ} \mathrm{C}, 2 \mathrm{~min}$ at $72{ }^{\circ} \mathrm{C}$; 3 cycles of $30 \mathrm{sec}$ at $95^{\circ} \mathrm{C}, 45 \mathrm{sec}$ at $53^{\circ} \mathrm{C}, 2 \mathrm{~min}$ at $72^{\circ} \mathrm{C}$; 32 cycles of $30 \mathrm{sec}$ at $95^{\circ} \mathrm{C}, 45 \mathrm{sec}$ at $52^{\circ} \mathrm{C}, 2 \mathrm{~min}$ at $72{ }^{\circ} \mathrm{C}$ and a final extension step of $7 \mathrm{~min}$ at $72^{\circ} \mathrm{C}$. Amplification products were separated on $2.5 \%$ agarose gel containing $0.1 \%$ ethidium bromide in $1 \times$ TBE Buffer $(89 \mathrm{mM}$ Tris - borate, $2.5 \mathrm{mM}$ EDTA). DNA marker GeneRulerTM 100 bp Plus DNA Ladder was used.

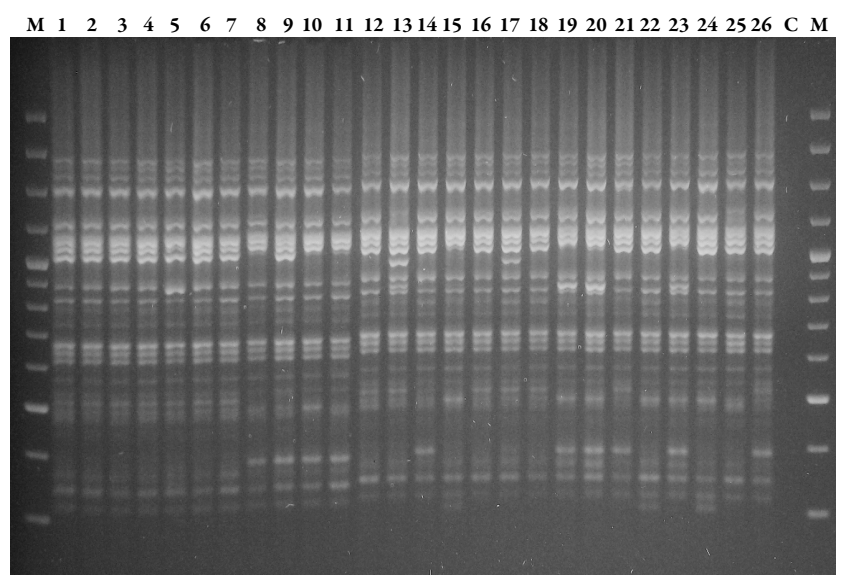

Fig. 1. An example of the DNA fragments profiling with primer SR - 55

M - 100bp Gene Ruler (Fermentas), 1 - 'Akt', 2 - 'Akt' 1, 3 - 'Akt' 2, 4 - 'Akt' bis, 5 - STH 721, 6 - 'Polar', 7 - 'Cacko', 8 - 'Maczo', 9 - STH 8307, 10 - STH 6315, 11 'Siwek', 12 - 'Nagus' 13 - DC 2188, 14 - DC 2215, 15 - DC 2612, 16 - DC 2711 , 17 - DC 2973, 18 - DC 3674, 19 - DC 3080, 20 - DC 3996, 21 - DC 3416, 22 DC 3249, 23 - DC 3170, 24 - DC 2567, 25 - DC 2931, 26 - DC 2934, C - negative control

\section{Data analysis}

The amplified ISSR fragments were scored as present (1) or absent $(0)$ and converted into a form of a binary matrix. Genetic pairwise similarities were evaluated using Nei's coefficient (Nei and Li, 1979). Polymorphic information content (PIC) values were estimated for each marker (Powell et al., 1996). UPGMA cluster analyses were performed using PAST software. The branches robustness was estimated using 1000 bootstrap replicates within PAST program. PCoA was performed in XIStat v.7.5.2.

\section{Results and discussions}

Although oat is a marginal cereal in Poland, by now, at least five naked domestic oats cultivars ('Polar', 'Cacko', 'Maczo', 'Siwek' and 'Nagus'), and many advanced lines have been tested by breeders. Naked forms of $A$. sativa seem to show promise for feeding and food production, but little is known on genetic variability of available cultivars and lines and their distinctiveness. This information is crucial for further breeding programs. Among many molecular marker systems useful for such studies, the ISSR approach has been used in wheat (Ammiraju et al., 2001), barley (Hou et al., 2005), maize (Carvalho et al., 2002) and rice (Bao et al., 2006; Qian et al., 2001). In all cases, the ISSR markers could identify as few as $34 \%$ and as many as $98 \%$ of polymorphisms useful for plant material differentiation.

In the given study, five cultivars and 18 advanced breeding lines of oat were tested with the ISSR method. Clearly visible and repeatable profiles were obtained (Fig. 1). Out of fifty ISSR primers screened for repeatable polymorphisms, 25 produced 429 scorable fragments, where 204 (47\%) were polymorphic (Tab. 2). The percentage of polymorphic bands is congruent with earlier data (Boczkowska and Tarczyk, 2013) - 59.3\% obtained for Polish oat landraces.

The number of fragments obtained for 26 analysed genotypes ranged from five (amplified by SR-27) to twenty six (SR-37 and SR-55), with an average 17.2 fragments per primer. Detected polymorphism varied from twenty (SR-27) to a hundred percent (SR-22) among primers. The number of polymorphic bands amplified by a single ISSR primer ranged from 1 (SR-27) to 15 (SR-23) with an average equal to 8.1 .

PIC characterizes the given marker system demonstrating its informativeness. It is usually assumed that the higher its value the better it fits experimental requirements with a value around 0.3 being sufficient for most taxonomic purposes (Botstein et al., 1980). PIC for the primers used in a given experiment ranged from 0.15 (SR-50) to 0.37 (SR-53) with an average equal to 0.23 per primer (Tab. 2). Previously, slightly lower values of PIC indices were obtained based on ISSRs (Boczkowska and Tarczyk, 2013) in case of Polish oat landraces. The average PIC for the particular marker ranged from 0.17 to 0.23 with the mean of 0.20 . Higher indices (0.26-0.40) were evaluated in A. fatua L. (Paczos-Grzeda $e t$ al., 2009). While PIC values evaluated by us were not too high, they were large enough to assume that ISSR marker system was capable of differentiating the analysed materials.

There were 44 markers unique to individual cultivars or lines (Tab. 2). Those markers constituted of $10.2 \%$ of the identified ISSR products. Out of all unique products recognized in this study, 21 were specific for the STH 721 line, that resulted from a cross between cv. 'Akt' and $A$. sterilis AVE 2999. The line specific markers originated from A. sterilis as they were not identified in cv. 'Akt'. Four line specific markers were amplified in cv. 'Maczo' and STH 6315, three in - DC 2188 and two in STH 8307, DC 2934 and DC 3416. Single unique amplicons were detected in 'Akt', DC 2612, DC 2711, DC 2931, DC 3996 and DC 3170. Twelve forms had no unique markers. Those cultivars and lines could be recognized by ISSRs fragment profiles amplified by one or more ISSR primers. 
Tab. 2. Characteristic of primers and detected polymorphism

\begin{tabular}{|c|c|c|c|c|c|c|c|}
\hline \multirow{2}{*}{ Primer } & \multirow{2}{*}{ Sequence $5^{\prime} \rightarrow 3^{\prime}$} & \multicolumn{4}{|c|}{ No of DNA fragments } & \multirow{2}{*}{$\begin{array}{c}\% \text { of } \\
\text { polymorphic } \\
\text { fragments }\end{array}$} & \multirow{2}{*}{ PIC } \\
\hline & & total & monomorphic & polymorphic & specific & & \\
\hline$S R-1$ & $(\mathrm{AG})_{8} \mathrm{G}$ & 19 & 14 & 5 & 1 & 26.3 & 0.189 \\
\hline$S R-11$ & $(\mathrm{AC})_{8} \mathrm{G}$ & 15 & 9 & 6 & 1 & 40.0 & 0.280 \\
\hline SR -17 & $(\mathrm{GA})_{8} \mathrm{C}$ & 23 & 13 & 10 & 0 & 43.5 & 0.260 \\
\hline$S R-22$ & $(\mathrm{CA})_{8} \mathrm{G}$ & 6 & 0 & 6 & 2 & 100.0 & 0.108 \\
\hline$S R-27$ & $(\mathrm{TC})_{8} \mathrm{G}$ & 5 & 4 & 1 & 0 & 20.0 & 0.210 \\
\hline$S R-28$ & $(\mathrm{TG})_{8} \mathrm{G}$ & 19 & 12 & 7 & 0 & 36.8 & 0.402 \\
\hline$S R-33$ & $(\mathrm{AG})_{8} \mathrm{~T}$ & 21 & 9 & 12 & 3 & 57.1 & 0.266 \\
\hline$S R-37$ & $(\mathrm{AC})_{8} \mathrm{C}$ & 26 & 15 & 11 & 2 & 42.3 & 0.155 \\
\hline$S R-39$ & $(\mathrm{GA})_{8} \mathrm{GG}$ & 19 & 10 & 9 & 4 & 47.4 & 0.180 \\
\hline$S R-40$ & $(\mathrm{AC})_{8} \mathrm{~T}$ & 20 & 15 & 5 & 1 & 25.0 & 0.243 \\
\hline$S R-42$ & $(\mathrm{AG})_{8} \mathrm{YA}$ & 20 & 7 & 13 & 2 & 65.0 & 0.268 \\
\hline$S R-45$ & $(\mathrm{GA})_{8} \mathrm{~T}$ & 15 & 7 & 8 & 0 & 53.3 & 0.260 \\
\hline$S R-46$ & $(\mathrm{GA})_{10} \mathrm{~A}$ & 19 & 5 & 14 & 6 & 73.7 & 0.164 \\
\hline$S R-48$ & $(\mathrm{CA})_{10} \mathrm{~T}$ & 13 & 6 & 7 & 1 & 53.8 & 0.316 \\
\hline$S R-49$ & $(\mathrm{TC})_{9} \mathrm{~A}$ & 14 & 10 & 4 & 3 & 28.6 & 0.196 \\
\hline$S R-50$ & $(\mathrm{TC}){ }_{9} \mathrm{C}$ & 16 & 7 & 9 & 3 & 56.3 & 0.153 \\
\hline$S R-53$ & $(\mathrm{CT})_{8} \mathrm{~A}$ & 17 & 2 & 15 & 2 & 88.2 & 0.368 \\
\hline$S R-55$ & $(\mathrm{CAC})_{6} \mathrm{~A}$ & 26 & 12 & 14 & 2 & 53.8 & 0.236 \\
\hline$S R-56$ & $(\mathrm{CAC})_{6} \mathrm{G}$ & 13 & 10 & 3 & 0 & 23.1 & 0.235 \\
\hline$S R-57$ & $(\mathrm{CTC})_{6} \mathrm{CG}$ & 13 & 8 & 5 & 2 & 38.5 & 0.189 \\
\hline$S R-58$ & $(\mathrm{ACC})_{6} \mathrm{~T}$ & 20 & 11 & 9 & 2 & 45.0 & 0.228 \\
\hline$S R-61$ & $(\mathrm{CAC})_{6} \mathrm{G}$ & 20 & 12 & 8 & 3 & 40.0 & 0.177 \\
\hline$S R-65$ & $(\mathrm{ATG})_{6} \mathrm{~T}$ & 13 & 6 & 7 & 0 & 53.8 & 0.278 \\
\hline$S R-69$ & $(\mathrm{AC})_{7} \mathrm{G}$ & 19 & 11 & 8 & 3 & 42.1 & 0.157 \\
\hline$S R-70$ & $(\mathrm{AC})_{8} \mathrm{YG}$ & 18 & 12 & 6 & 1 & 33.3 & 0.207 \\
\hline Total & & 429 & 227 & 204 & 44 & & \\
\hline Average & & 17.2 & 9.1 & 8.1 & 1.8 & 47.5 & 0.229 \\
\hline
\end{tabular}

Tab 3. ISSR markers number differentiating naked oat cultivars and lines

\begin{tabular}{|c|c|c|c|c|c|c|c|c|c|c|c|c|c|c|c|c|c|c|c|c|c|c|c|c|c|}
\hline $\begin{array}{l}\text { Cultivar/ } \\
\text { Line }\end{array}$ & $\frac{3}{3}$ & $\vec{z}$ & 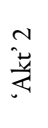 & $\begin{array}{l}\frac{a}{0} \\
\frac{\vec{y}}{4}\end{array}$ & $\begin{array}{l}\vec{N} \\
\stackrel{\vec{N}}{\vec{I}} \\
\text { 点 }\end{array}$ & $\stackrel{2}{\frac{\pi}{0}}$ & 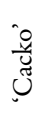 & 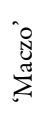 & 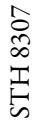 & 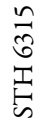 & 蒴 & 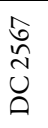 & 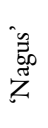 & $\begin{array}{l}\infty \\
\stackrel{\infty}{N} \\
\bigcup \\
ٍ\end{array}$ & 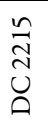 & 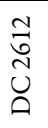 & 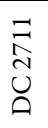 & 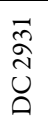 & 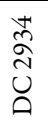 & $\begin{array}{l}\stackrel{N}{\hat{N}} \\
\stackrel{0}{0}\end{array}$ & 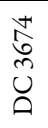 & 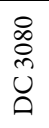 & $\begin{array}{l}\stackrel{2}{~} \\
\text { ঠे } \\
0\end{array}$ & 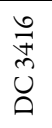 & 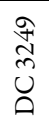 \\
\hline 'Akt' 1 & 3 & & & & & & & & & & & & & & & & & & & & & & & & \\
\hline 'Akt' 2 & 4 & 3 & & & & & & & & & & & & & & & & & & & & & & & \\
\hline 'Akt' bis & 1 & 2 & 3 & & & & & & & & & & & & & & & & & & & & & & \\
\hline STH 721 & 69 & 68 & 69 & 68 & & & & & & & & & & & & & & & & & & & & & \\
\hline 'Polar' & 31 & 30 & 31 & 30 & 78 & & & & & & & & & & & & & & & & & & & & \\
\hline 'Cacko' & 35 & 34 & 35 & 34 & 76 & 8 & & & & & & & & & & & & & & & & & & & \\
\hline 'Maczo' & 56 & 55 & 54 & 55 & 91 & 55 & 61 & & & & & & & & & & & & & & & & & & \\
\hline STH 8307 & 61 & 62 & 63 & 60 & 92 & 54 & 56 & 61 & & & & & & & & & & & & & & & & & \\
\hline STH 6315 & 62 & 63 & 64 & 61 & 95 & 65 & 67 & 50 & 59 & & & & & & & & & & & & & & & & \\
\hline 'Siwek' & 23 & 24 & 25 & 22 & 78 & 43 & 48 & 66 & 58 & 65 & & & & & & & & & & & & & & & \\
\hline DC 2567 & 38 & 39 & 40 & 37 & 83 & 41 & 45 & 58 & 63 & 54 & 47 & & & & & & & & & & & & & & \\
\hline 'Nagus' & 31 & 32 & 33 & 30 & 74 & 40 & 38 & 63 & 60 & 67 & 42 & 25 & & & & & & & & & & & & & \\
\hline DC 2188 & 56 & 57 & 58 & 55 & 89 & 53 & 55 & 70 & 51 & 70 & 65 & 62 & 57 & & & & & & & & & & & & \\
\hline DC 2215 & 46 & 47 & 48 & 45 & 87 & 45 & 43 & 62 & 57 & 64 & 41 & 46 & 49 & 66 & & & & & & & & & & & \\
\hline DC 2612 & 49 & 50 & 49 & 48 & 86 & 44 & 42 & 65 & 68 & 65 & 56 & 39 & 36 & 63 & 47 & & & & & & & & & & \\
\hline DC 2711 & 24 & 23 & 24 & 23 & 67 & 27 & 27 & 56 & 55 & 64 & 37 & 42 & 35 & 54 & 44 & 47 & & & & & & & & & \\
\hline DC 2931 & 37 & 38 & 39 & 36 & 84 & 24 & 30 & 59 & 60 & 71 & 48 & 33 & 42 & 53 & 45 & 52 & 39 & & & & & & & & \\
\hline DC 2934 & 37 & 38 & 37 & 36 & 82 & 28 & 36 & 53 & 56 & 65 & 46 & 39 & 42 & 57 & 45 & 52 & 35 & 20 & & & & & & & \\
\hline DC 2973 & 34 & 33 & 34 & 33 & 71 & 19 & 25 & 62 & 53 & 68 & 45 & 44 & 41 & 50 & 48 & 43 & 28 & 33 & 33 & & & & & & \\
\hline DC 3674 & 29 & 28 & 29 & 28 & 74 & 10 & 14 & 59 & 58 & 67 & 44 & 43 & 36 & 55 & 49 & 44 & 25 & 28 & 28 & 17 & & & & & \\
\hline DC 3080 & 57 & 58 & 59 & 56 & 92 & 62 & 64 & 71 & 82 & 57 & 66 & 55 & 64 & 73 & 71 & 60 & 59 & 62 & 56 & 61 & 60 & & & & \\
\hline DC 3996 & 56 & 57 & 58 & 55 & 93 & 63 & 65 & 72 & 81 & 58 & 65 & 56 & 65 & 72 & 72 & 59 & 60 & 63 & 57 & 62 & 61 & 3 & & & \\
\hline DC 3416 & 31 & 32 & 33 & 30 & 78 & 40 & 42 & 53 & 56 & 65 & 46 & 43 & 44 & 57 & 47 & 52 & 35 & 32 & 24 & 37 & 40 & 62 & 61 & & \\
\hline DC 3249 & 49 & 50 & 51 & 48 & 86 & 46 & 44 & 67 & 66 & 63 & 54 & 19 & 30 & 63 & 45 & 28 & 45 & 40 & 42 & 43 & 44 & 56 & 57 & 46 & \\
\hline DC 3170 & 60 & 61 & 62 & 59 & 93 & 65 & 67 & 68 & 85 & 60 & 67 & 56 & 67 & 74 & 64 & 63 & 64 & 61 & 55 & 66 & 63 & 15 & 16 & 61 & 61 \\
\hline
\end{tabular}


70

The lines and cultivars differed from each other by as few as one ('Akt' vs. 'Akt' bis) and as many as 95 markers (STH 6315 vs. STH 721) with the average number equal to fifty (Tab. 3). Lines representing cv. 'Akt' were distinguished from each other by four markers. Only three ISSRs discriminated the DC 3080 and 3996 lines while eight once 'Polar' and 'Cacko' cultivars. Ten markers distinguished the DC 3674 line and cultivar 'Polar' while 14 once from 'Cacko'. Some markers differentiated the DC 3170 and DC 3996 (15 markers), DC 3170 and DC 3416 (16), DC 3674 and DC 2973 (17), DC 3674/02 and
'Polar', DC $3249 / 02$ and DC 2567 (19). The DC 2931 and DC 2934, DC 1368 and DC 2567 were distinguished by 20 and 25 markers, respectively. 'Siwek' that originated from a cross between 'Benquel' and 'Akt', had 25 markers discriminating it from 'Akt'. The highest number of markers (from 67 to 95) differentiated line STH 721 ('Akt' $\mathrm{x} A$. sterilis AVE 229) from the other materials because it originated from an interspecific cross of $A$. sativa and $A$. sterilis. The latter is a wild relative of $A$. sativa and is being used as a source of valuable traits for oat improvement (Forsberg and Reeves, 1992).

Tab 4. Dice genetic similarity coefficients evaluated based on ISSR polymorphism

\begin{tabular}{|c|c|c|c|c|c|c|c|c|c|c|c|c|c|c|c|c|c|c|c|c|c|c|c|c|c|}
\hline $\begin{array}{l}\text { Cultivar/ } \\
\text { Line }\end{array}$ & $\vec{z}$ & $\vec{y}$ & $\begin{array}{l}n \\
\vec{z}\end{array}$ & $\frac{\frac{m}{3}}{\frac{\vec{y}}{3}}$ & $\begin{array}{l}\vec{N} \\
\underline{I} \\
\vec{\omega}\end{array}$ & $\stackrel{\pi}{0}$ & 总 & i & 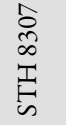 & 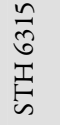 & 荡 & 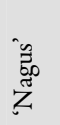 & 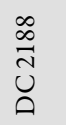 & 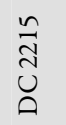 & 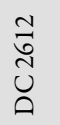 & $\begin{array}{l}\bar{N} \\
\text { U. } \\
0\end{array}$ & $\begin{array}{l}\hat{N} \\
\hat{N} \\
0\end{array}$ & 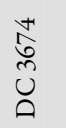 & 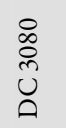 & $\begin{array}{l}\text { ूे } \\
\text { Uे } \\
0\end{array}$ & 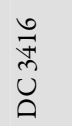 & $\begin{array}{l}\text { Jे } \\
\text { U్ } \\
\text { }\end{array}$ & $\begin{array}{l}\stackrel{尺}{0} \\
\hat{0} \\
0\end{array}$ & 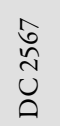 & $\begin{array}{l}\overline{\widetilde{N}} \\
\text { Ù }\end{array}$ \\
\hline 'Akt' 1 & 0.98 & & & & & & & & & & & & & & & & & & & & & & & & \\
\hline 'Akt' 2 & 0.97 & 0.98 & & & & & & & & & & & & & & & & & & & & & & & \\
\hline 'Akt' bis & 0.99 & 0.99 & 0.98 & & & & & & & & & & & & & & & & & & & & & & \\
\hline STH 721 & 0.56 & 0.56 & 0.56 & 0.56 & & & & & & & & & & & & & & & & & & & & & \\
\hline 'Polar' & 0.77 & 0.78 & 0.77 & 0.78 & 0.49 & & & & & & & & & & & & & & & & & & & & \\
\hline 'Cacko' & 0.75 & 0.75 & 0.75 & 0.75 & 0.50 & 0.94 & & & & & & & & & & & & & & & & & & & \\
\hline 'Maczo' & 0.62 & 0.63 & 0.63 & 0.62 & 0.44 & 0.62 & 0.57 & & & & & & & & & & & & & & & & & & \\
\hline STH 8307 & 0.58 & 0.58 & 0.57 & 0.58 & 0.43 & 0.62 & 0.61 & 0.60 & & & & & & & & & & & & & & & & & \\
\hline STH 6315 & 0.58 & 0.58 & 0.57 & 0.59 & 0.42 & 0.55 & 0.54 & 0.68 & 0.61 & & & & & & & & & & & & & & & & \\
\hline 'Siwek' & 0.84 & 0.83 & 0.82 & 0.84 & 0.49 & 0.68 & 0.65 & 0.55 & 0.60 & 0.56 & & & & & & & & & & & & & & & \\
\hline 'Nagus' & 0.77 & 0.77 & 0.76 & 0.78 & 0.51 & 0.70 & 0.71 & 0.55 & 0.57 & 0.53 & 0.69 & & & & & & & & & & & & & & \\
\hline DC 2188 & 0.61 & 0.60 & 0.59 & 0.61 & 0.43 & 0.62 & 0.60 & 0.53 & 0.65 & 0.53 & 0.54 & 0.58 & & & & & & & & & & & & & \\
\hline DC 2215 & 0.67 & 0.66 & 0.65 & 0.67 & 0.43 & 0.67 & 0.68 & 0.57 & 0.60 & 0.56 & 0.70 & 0.63 & 0.53 & & & & & & & & & & & & \\
\hline DC 2612 & 0.64 & 0.63 & 0.64 & 0.64 & 0.43 & 0.67 & 0.68 & 0.54 & 0.51 & 0.55 & 0.58 & 0.72 & 0.54 & 0.65 & & & & & & & & & & & \\
\hline DC 2711 & 0.83 & 0.84 & 0.83 & 0.84 & 0.58 & 0.81 & 0.81 & 0.63 & 0.63 & 0.58 & 0.74 & 0.75 & 0.63 & 0.69 & 0.66 & & & & & & & & & & \\
\hline DC 2973 & 0.76 & 0.77 & 0.76 & 0.76 & 0.54 & 0.86 & 0.82 & 0.58 & 0.63 & 0.54 & 0.68 & 0.70 & 0.65 & 0.65 & 0.68 & 0.81 & & & & & & & & & \\
\hline DC 3674 & 0.79 & 0.80 & 0.79 & 0.80 & 0.53 & 0.93 & 0.90 & 0.60 & 0.60 & 0.55 & 0.69 & 0.74 & 0.62 & 0.65 & 0.68 & 0.83 & 0.88 & & & & & & & & \\
\hline DC 3080 & 0.63 & 0.62 & 0.61 & 0.63 & 0.45 & 0.59 & 0.57 & 0.55 & 0.48 & 0.65 & 0.57 & 0.57 & 0.53 & 0.53 & 0.60 & 0.62 & 0.60 & 0.61 & & & & & & & \\
\hline DC 3996 & 0.64 & 0.63 & 0.62 & 0.64 & 0.45 & 0.58 & 0.57 & 0.55 & 0.49 & 0.64 & 0.58 & 0.56 & 0.54 & 0.53 & 0.60 & 0.62 & 0.60 & 0.61 & 0.98 & & & & & & \\
\hline DC 3416 & 0.79 & 0.78 & 0.77 & 0.79 & 0.51 & 0.72 & 0.70 & 0.65 & 0.63 & 0.58 & 0.68 & 0.69 & 0.61 & 0.67 & 0.63 & 0.77 & 0.75 & 0.73 & 0.61 & 0.62 & & & & & \\
\hline DC 3249 & 0.65 & 0.64 & 0.63 & 0.65 & 0.44 & 0.66 & 0.68 & 0.54 & 0.54 & 0.57 & 0.61 & 0.78 & 0.55 & 0.67 & 0.79 & 0.69 & 0.69 & 0.69 & 0.63 & 0.63 & 0.68 & & & & \\
\hline DC 3170 & 0.60 & 0.60 & 0.59 & 0.60 & 0.44 & 0.56 & 0.54 & 0.56 & 0.45 & 0.62 & 0.55 & 0.54 & 0.51 & 0.57 & 0.57 & 0.58 & 0.56 & 0.58 & 0.91 & 0.90 & 0.61 & 0.59 & & & \\
\hline DC 2567 & 0.73 & 0.72 & 0.71 & 0.73 & 0.46 & 0.70 & 0.67 & 0.60 & 0.56 & 0.63 & 0.66 & 0.81 & 0.56 & 0.66 & 0.71 & 0.70 & 0.68 & 0.69 & 0.64 & 0.63 & 0.70 & 0.86 & 0.62 & & \\
\hline DC 2931 & 0.73 & 0.73 & 0.72 & 0.74 & 0.45 & 0.82 & 0.78 & 0.59 & 0.58 & 0.51 & 0.65 & 0.68 & 0.62 & 0.67 & 0.61 & 0.72 & 0.76 & 0.80 & 0.59 & 0.58 & 0.78 & 0.71 & 0.59 & 0.76 & \\
\hline DC 2934 & 0.74 & 0.74 & 0.74 & 0.75 & 0.48 & 0.80 & 0.74 & 0.64 & 0.62 & 0.57 & 0.68 & 0.70 & 0.61 & 0.68 & 0.62 & 0.76 & 0.77 & 0.81 & 0.64 & 0.64 & 0.84 & 0.70 & 0.64 & 0.72 & 0.86 \\
\hline
\end{tabular}

The genetic similarity matrix was obtained based on ISSR data using the Dice's coefficient (Tab. 4). The lowest value of genetic similarity was observed for the STH 721 and STH 6315 lines (0.42), whereas the highest for 'Akt' and 'Akt' bis (0.99). The mean genetic similarity was 0.65 . Very high genetic similarity coefficients were also found between cv. 'Akt' and its sister lines (0.98), cv. 'Polar' and 'Cacko' (0.94) as well as DC 3674 line and cv. 'Polar' (0.93). The observed high level of similarity among 'Akt' and its sister lines demonstrated that ISSR markers are capable of identifying even very few differences that may exhibit some minor variation within the above mentioned materials. Thus, cv. 'Akt' and its sublines are identical. In this context, it seems surprising that a high similarity of some cultivars do not have 'Akt' in its crossing formulas with 'Akt' may suggest mistakes during crossing or incorrect pedigree data.

While UPGMA failed to distinguish the materials into main clusters it demonstrated that cultivars 'Akt', 'Polar', 'Cacko', 'Siwek', 'Nagus' and most of the DC lines were within a single group. Moreover, the cultivars that were closely related based on their breeding pedigree (namely, those related to 'Akt'), were close to each other. Also three DC lines (DC 3080; DC 3996; DC 3170) that originated from the same cross formula $(\mathrm{P}(508) 19251 \times \mathrm{CHD} 1743)$ were closely related. High level of similarity was observed between two lines DC 3080 and DC 3996. Although breeding pedigree between cultivar 'Maczo' and STH 6315 line, as well as STH 8307 and DC 2188 lines, is not known, the materials appeared to be relatively close to each other. In general, the DC lines have lower variation than the STH pool. Interestingly, that similar analyses performed on 23 old cultivars using AFLP, ISSR and RAPD markers failed in any significant grouping of the analysed materials (Boczkowska et al., 2014). The data demonstrates that the analysed materials show enough variation useful for breeding purposes.

Interestingly, that STH 721 line located at the edge of the dendrogram (Fig. 2). It originated from a cross where $A$. 
sterilis was a counterpart. This is the only such line used in the study. While the STH 721 is an advanced line, evidently, such grouping could be explained by the presence of $A$. sterilis DNA that was not eliminated despite years of selection.

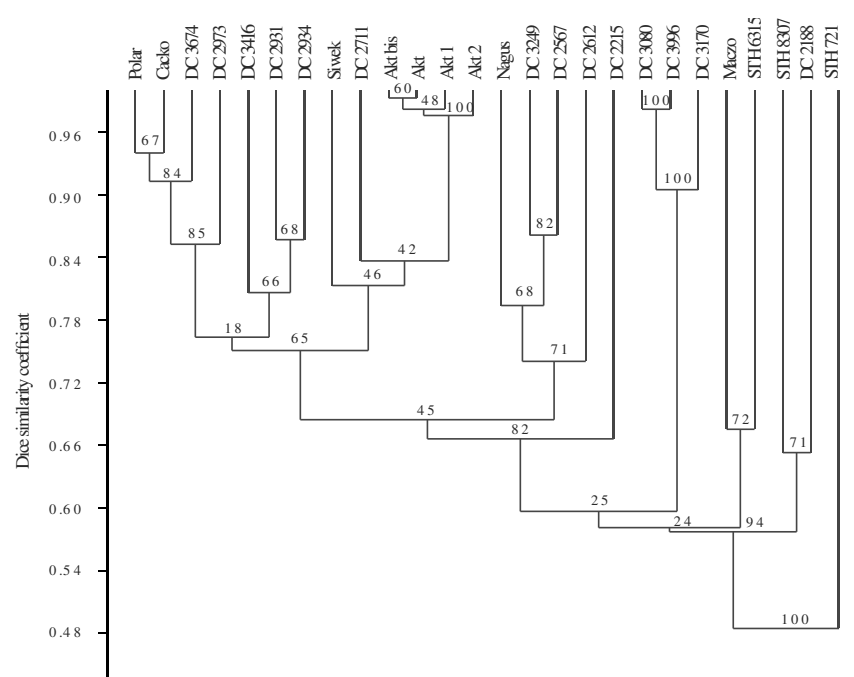

Fig 2. Dendrogram representing 26 oat cultivars and lines. UPGMA method and Dice similarity index evaluated on ISSR markers were used

In general, Principal Coordinate Analysis was in good agreement with the UPGMA (Fig. 3). The first two main components of PCoA explained $54.1 \%$ of the variance and allowed partial differentiation of the materials. Evidently, DC 3170 , DC 3080 and DC 3996 lines originated from the same cross formula $(\mathrm{P}(508) 19251 \mathrm{x}$ CHD 1743) established a separate group. Similarly DC 2567, DC 2612 and DC 3249 that have similar parental forms made the second one which was close to the group of cultivars related to 'Akt' as well as to the group of materials encompassing cv. 'Cacko', 'Polar' and some DC lines that have 'Polar' or 'Sallust' in pedigrees. As in the case of the UPGMA, the STH 721 line was separated from all the materials.

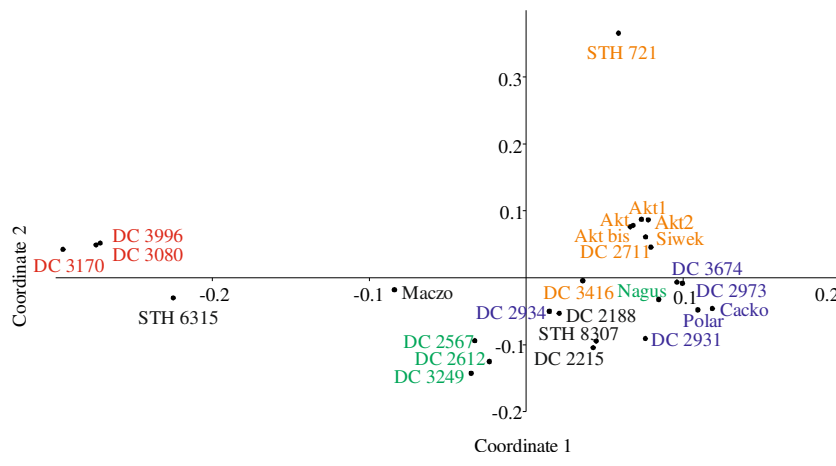

Fig 3. Principle component analyses of oat cultivars and lines originated from different breeding companies and evaluated based on ISSR markers. 'Polar', 'Cacko' and DC lines with 'Polar' or 'Sallust' in pedigrees are indicated in blue; the DC lines that have common origin are given in red; cv. 'Akt' and genotypes with 'Akt' in their pedigrees are depicted in orange; cv. 'Nagus' and lines with similar parental forms are indicated in green

\section{Conclusions}

Based on the current study it was demonstrated that ISSR markers could be used for the evaluation of available genetic pool of naked $A$. sativa lines and cultivars and differentiation of individual genotypes. Such approach seems to be a method of choice when limited funds are available. Moreover, the ISSR markers are cheap, cost effective and highly reproducible comparative to e.g. SNP markers. Evidently, the method could be successfully applied for expended analyses of oat accessions or genotype identification.

The presented results demonstrate that Polish breeding materials represent relatively rich genetic pool where the most variable, are lines that are currently under preliminary field experiments. According to our data, the naked oat genetic pool available for breeders, could be efficiently exploited in the future to create new cultivars.

\section{References}

Ammiraju JSS, Dholakia BB, Santra DK, Singh H, Lagu MD, Tamhankar SA, Dhaliwal HS, Rao VS, Gupta VS, Ranjekar PK (2001). Identification of inter simple sequence repeat (ISSR) markers associated with seed size in wheat. Theor Appl Genet 102:726-732.

Bao J, Corke H, Sun M (2006). Analysis of genetic diversity and relationships in waxy rice (Oryza sativa L.) using AFLP and ISSR markers. Genet Resour Crop Ev 53:323-330.

Boczkowska M, Nowosielski J, Nowosielska D, Podyma W (2014). Assessing genetic diversity in 23 early Polish oat cultivars base on molecular and morphological studies. Genet Resour Crop Ev DOI 101007/s10722-014-0087-4.

Boczkowska M, Tarczyk E (2013). Genetic diversity among Polish landraces of common oat (Avena sativa L.). Genet Resour Crop Ev 60:2157-2169.

Botstein D, White RL, Skolnick M, Davis RW (1980). Constructions of genetic linkage map in man using restriction fragment length polymorphisms. Am J Hum Genet 32:314333.

Burrows VD (1986). Breeding oats for food and feed: conventional and new techniques and materials. 13-46 p. In: Webster FH (Ed.). Oats: chemistry and technology. St. Paul, Minnesota, USA.

Carvalho VP, Ruas PM, Ruas CF, Ferreira JM, Moreira RMP (2002). Assessment of genetic diversity in maize (Zea mays L.) landraces using inter simple sequence repeat (ISSR) markers. Crop Breed Appl Biot 2:557-568.

Chowdhury MA, Vandenberg B, Warkentin T (2000). Cultivar identification and genetic relationship among selected breeding lines and cultivars in chickpea (Cicer arietinum L.). Euphytica 127:317-325.

Cox TS, Murphy JP (1990). The effect of parental divergence on $\mathrm{F}_{2}$ heterosis in winter wheat crosses. Theor Appl Genet 79:241-50. 
72

Doehlert DC, Jannink JL, McMullen MS (2006). Kernel size variation in naked oat. Crop Sci. 46:1117-1123.

Doyle JJ, Doyle JL (1987). A rapid DNA isolation procedure for small quantities of fresh leaf tissue. Phytochemistry Bulletin 19:11-15.

Forsberg RA, Reeves DL (1992). Breeding oat cultivars for improved grain quality. 751-775 p. In: Sorrells ME, Marshall HG (Eds.). Oat Science and Technology. American Society of Agronomy. Agronomy Monograph No.33, Madison, Wis., USA.

Fu YB, Peterson GW, Williams D, Richards KW, Fetch JM (2005). Patterns of AFLP variation in a core subset of cultivated hexaploid oat germplasm. Theor Appl Genet 11:530-9.

Hou YC, Yan ZH, Wei YM, Zheng YL (2005). Genetic diversity in barley from west China based on RAPD and ISSR analysis. Barley Genetics Newsletter 35:9-22.

Kirkkari AM (2008). Comparative economic analysis for production of naked vs. conventional oat. Acta Agr Scand 58:305-313.

Kirkkari AM, Peltonen-Sainio P, Jauhiainen L (2009). Trichome numbers in naked-oat cultivars. J Agr Sci 147:63-69.

Martin JM, Blake TK, Hockett EA (1991). Diversity among North American spring barley cultivars based on coefficients of parentage. Crop Sci 31:1131-1137.

Nei M, Li WH (1979). Mathematical model for studying genetic variation in terms of restriction endonucleases. P Natl Acad Sci USA 76:5269-73.

Nita ZT (2003). Contemporary achievements and prospects of oat breeding in Poland. Biuletyn IHAR 229: 13-20 (in Polish with abstract in English).

Paczos-Grzeda E (2004). Pedigree, RAPD and simplified AFLPbased assessment of genetic relationship among Avena sativa L. cultivars. Euphytica 138:13-22.

Paczos-Grzeda E, Kruk K, Okon S (2009). Assessment of Avena fatua L. intraspecific genetic similarity based on DNA polymorphism identified by ISSR and REMAP Methods. Biuletyn IHAR 252:235-243 (in Polish with abstract in English).
Powell W, Morgante M, Andre C, Hanafey M (1996). Comparison of RFLP, RAPD, AFLP and SSR (Microsatellite) markers for germplasm analysis. Mol Breeding 2:225-238.

Qian W, Ge S, Hong DY (2001). Genetic variation within and among populations of a wild rice Oryza granulata from China detected by RAPD and ISSR markers. Theor Appl Genet 102:440-449.

Ronald PS, Brown PD, Penner GA, Brûlé-Babel A, Kibite S (1999). Heritability of hull percentage in oat. Crop Sci 39:5257.

Ronald PS, Penner GA, Brown PD, Brule-Babel A (1997). Identification of RAPD markers for percent hull in oat. Genome 40:873-8.

Salo M-L, Kotilainen K (1970). On the carbohydrate composition and nutritive value of some cereals. J Agr Sci Finland 42:21-29.

Sykut-Domańska E (2012). Characterisation of selected physical properties of naked and common oat (Avena sativa L.) Acta Agrophysica 19:845-856.

Tinker NA, Kilian A, Wight CP, Heller-Uszynska K, Wenzl P, Rines HW, Bjornstad A, Howarth CJ, Jannink JL, Anderson JM, Rossnagel BG, Stuthman DD, Sorrells ME, Jackson EW, Tuvesson S, Kolb FL, Olsson O, Federizzi LC, Carson ML, Ohm HW, Molnar SJ, Scoles GJ, Eckstein PE, Bonman JM, Ceplitis A, Langdon T (2009). New DArT markers for oat provide enhanced map coverage and global germplasm characterization. BMC Genomics 10: 39.

Valentine J (1995). Naked oats. 504-532 p. In: Welch RW (Ed.). The Oat Crop: Production and Utilization. Chapman \& Hall, England.

Welch R, Hayward MV, Jones DIH (1983). The composition of the oat husk and its variation due to genetic and other factors. Cereal Chem 34:417-426.

Zeller FJ (1998). Nutzung Des Genetischen Potentials Der Avena - Wildarten Zur Verbesserung Des Saathafers (Avena sativa L.). J Appl Bot 72: 180-185 (in German).

Zietkiewicz E, Rafalski A, Labuda D (1994). Genome fingerprinting by simple sequence repeat (SSR)-anchored polymerase chain reaction amplification. Genomics 20:17683. 